\title{
Pólipo y leiomioma uterinos en un erizo pigmeo africano (Atelerix albiventris)
}

\author{
Uterine polyp and leiomyoma in an African pigmy hedgehog (Atelerix \\ albiventris)
}

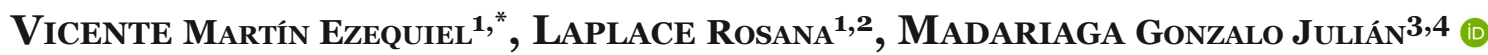 \\ García MitaceK María CARLA ${ }^{2,5}$
}

1. Servicio de Especies No Tradicionales del Hospital Escuela. 2. Cátedra y Servicio de Reproducción Animal. 3. Laboratorio de Patología Especial Veterinaria "Dr. B. Epstein". 4. Cátedra de Clínica de Pequeños Animales. Facultad de Ciencias Veterinarias. Universidad Nacional de La Plata. 5. Consejo Nacional de Investigaciones Científicas y Técnicas (CONICET).

* Correo electrónico del autor de contacto: mvicente@fcv.unlp.edu.ar

\begin{abstract}
Resumen
El erizo pigmeo africano (Atelerix albiventris) se ha convertido en una mascota exótica. Es por ello que se han realizado diversos estudios sobre aspectos de salud y enfermedad de esta especie. El objetivo del trabajo fue presentar un caso de un pólipo y leiomioma uterinos en un erizo pigmeo africano. En 2016, una hembra de 2 años se presentó a la consulta con hematuria. La radiografía y ecografía abdominales revelaron una masa intrauterina. Se realizó ovariohisterectomía y se constató la presencia de dos masas de tejido. Mediante estudios histopatológico e histoquímico se formuló un diagnóstico de pólipo y leiomioma uterinos. La paciente se recuperó totalmente. Doce meses postintervención la misma concurrió a consulta para realizar un control anual en el cual se no se evidenciaron signos clínicos de la enfermedad.
\end{abstract}

\section{Palabras clave}

Atelerix albiventris, pólipo endometrial, leiomioma uterino

\begin{abstract}
The African pigmy hedgehog (Atelerix albiventris) has become an exotic pet. Therefore, interest on health and disease aspects of this species has increased. The aim of this study was to describe a case of uterine polyp and leiomyoma in an African pigmy hedgehog. In 2016, a 2 years old female came to the clinic presenting hematuria. Abdominal radiography and ultrasound were performed and revealed an intrauterine mass. An ovariohysterectomy was performed and the presence of two tissue masses was observed. histopathological and histochemical analysis of the neoformations confirmed the diagnosis of uterine polyp and leiomyoma. The patient recovered completely. Twelve months post-intervention, the animal was clinically evaluated at an annual control in which clinical signs of the disease were not evidenced.
\end{abstract}

\section{Key words}

Atelerix albiventris, endometrial polyp, uterine leiomyoma

Fecha de recepción: 29/03/2019

Fecha de revisión: 13/08/2019

Fecha de aprobación: 13/09/2019
ANALECTA Vet 2019; Julio-Diciembre; 39(2):47-51

Impresa ISSN 03655 14-8 Electrónica ISSN 1514-2590

doi.org/10.24215/15142590e042 


\section{Introducción}

El erizo pigmeo africano (Atelerix albiventris) es un mamífero del orden Erinaceomorpha de la familia Erinaceidae, subfamilia Erinaceinae. Nativo de África ecuatorial, habita estepas, sabanas, pastizales y campos agrícolas. En los últimos años ha sido domesticado, convirtiéndose en una mascota exótica. Esta especie alcanza la madurez sexual, en estado salvaje, alrededor del año de edad. Atelerix albiventris se reproduce bien en cautiverio, siendo sexualmente activo durante todo el año sin aparente temporada de apareamiento (Santana et al., 2010). La esperanza de vida es de 1 a 4 años en estado silvestre. Sin embargo, con un buen asesoramiento nutricional y atención veterinaria adecuada ha ampliado su expectativa de vida en cautiverio (Heatley et al., 2005; Santana et al., 2010). La prevalencia de tumores en el erizo parece no estar relacionada con el sexo. Se ha comunicado un aumento de afecciones neoplásicas en erizos de más de 3 años de edad (Heatley et al., 2005; Raymond \& Garner, 2001). Generalemente, un $85 \%$ de los tumores son malignos. Los sistemas más frecuentemente involucrados incluyen el tegumentario, hemolinfático, digestivo, endocrino y reproductivo. Las neoplasias epiteliales son las más frecuentes, seguidas de las de células redondas y de células mesenquimáticas (Heatley et al., 2005; Raymond \& Garner, 2001). En las hembras, la neoplasia maligna más comúnmente encontrada es el adenosarcoma, seguido por el sarcoma estromal y el leiomiosarcoma. Generalmente, la edad de presentación es entre 2 y 4 años (Heatley et al., 2005; Mikaelian \& Reavill, 2004). Los signos clínicos típicos son: sangrado vaginal, hematuria y pérdida de peso. También los pólipos endometriales ulcerados o con necrosis pueden cursar con signos clínicos similares (Phillips et al., 2005). La detección precoz con posterior tratamiento mejora el pronóstico a largo plazo en animales afectados (Heatley et al., 2005; Mikaelian \& Reavill, 2004).

El pólipo endometrial ha sido descrito en humanos, chimpancés, perros, gatos, caballos, elefantes, perros salvajes africanos, ratones y erizos, entre otras especies. El mismo se caracteriza por una protrusión nodular del endometrio constituido por tejido conectivo, estroma fibroso y glándulas dilatadas, pudiendo generar distensión segmentaria de los cuernos uterinos. Los pólipos pueden ser únicos o múltiples (Chambers et al., 2018; McEntee \& Nielsen, 1976; Schlafer \& Miller, 2007).

Los leiomiomas son tumores benignos y han sido descriptos en la vaca, perra, gata y erizo, entre otras especies. Se originan en el músculo liso del útero, cuello uterino o vagina y pueden ser únicos o múltiples. Por lo general, los tumores más pequeños son blandos, pero al aumentar de tamaño pueden adquirir una consistencia firme o dura. Los leiomiomas pueden ubicarse en el miometrio o proyectarse como masas redondeadas u ovaladas hacia el lumen de la vagina, útero o cuello uterino. Histológicamente, el tumor está compuesto por haces de fibras musculares lisas arremolinadas y escaso tejido conectivo (McEntee \& Nielsen, 1976; Schlafer \& Miller, 2007). El tratamiento de elección es la ovariohisterectomía. En la actualidad no existen datos sobre tratamiento médico en el erizo pigmeo africano (Heatley et al., 2005; Mikaelian \& Reavill, 2004). En la especie humana, los tratamientos médicos se instauran cuando se quiere preservar la fertilidad y evitar o retrasar la cirugía. Actualmente, los agonistas de la hormona liberadora de gonadotropinas (GnRH) y los moduladores selectivos del receptor de progesterona son las terapias médicas más efectivas. Por lo general, el pronóstico en la mujer luego de la escisión quirúrgica es bueno (Sohn et al., 2018).

Si bien se han comunicado varios trabajos en los cuales se registran neoplasias uterinas en el erizo pigmeo africano, hasta el momento no hemos encontrado ningún trabajo en el cual se haya comunicado la presentación en forma conjunta de un leiomioma con un pólipo endometrial. El objetivo del trabajo fue presentar un caso clínico de pólipo y leiomioma uterinos en una erizo pigmeo africano (Atelerix albiventris).

\section{Presentación del caso}

En el año 2016 se presentó a consulta un erizo pigmeo africano (Atelerix albiventris), hembra, de 2 años y 240 g de peso, con hematuria de 7 días de evolución. En el examen clínico se observó un estado general bueno y parámetros fisiológicos normales (frecuencia cardíaca de 230 [180-260], frecuencia respitatoria de 45 [25-50] y temperatura corporal de $\left.37^{\circ} \mathrm{C}\left[35,4-37^{\circ} \mathrm{C}\right]\right)$. En la radiografía abdominal se observó una vejiga pletórica sin urolitos radiopacos y aumento de la radiopacidad circunscripta en mesogastrio con desplazamiento de órganos vecinos, sugerente de masa (Figura 1A). En la ecografía abdominal se constató una masa intrauterina de $15,4 \mathrm{~mm}$ de diámetro, de bordes irregulares, hipoecoica, con ecotextura granular gruesa y heterogénea a expensas de múltiples áreas anecoicas (Figura 1B). Se indicó la ovariohisterectomía bajo anestesia inhalatoria. Se realizó un tratamiento postquirúrgico con enrofloxacina $5 \mathrm{mg} / \mathrm{kg} /$ día/V.O. durante 7 días y meloxicam $0,2 \mathrm{mg} / \mathrm{kg} /$ día/V.O. durante 3 días. Posteriormente, se seccionó el útero y se constató la presencia de dos masas, una de ellas pedunculada y la otra nodular. Se obtuvieron muestras de ambos cuernos uterinos y de las dos masas para histopatología. Las muestras se fijaron en formaldehído bufferado al $10 \%$, se procesaron mediante las técnicas de rutina y se colorearon con hematoxilina y eosina. En los cortes histológicos de ambos cuernos uterinos se observó la presencia de un infiltrado mononuclear compuesto principalmente por macrófagos y edema en el endometrio (Figura 2). 


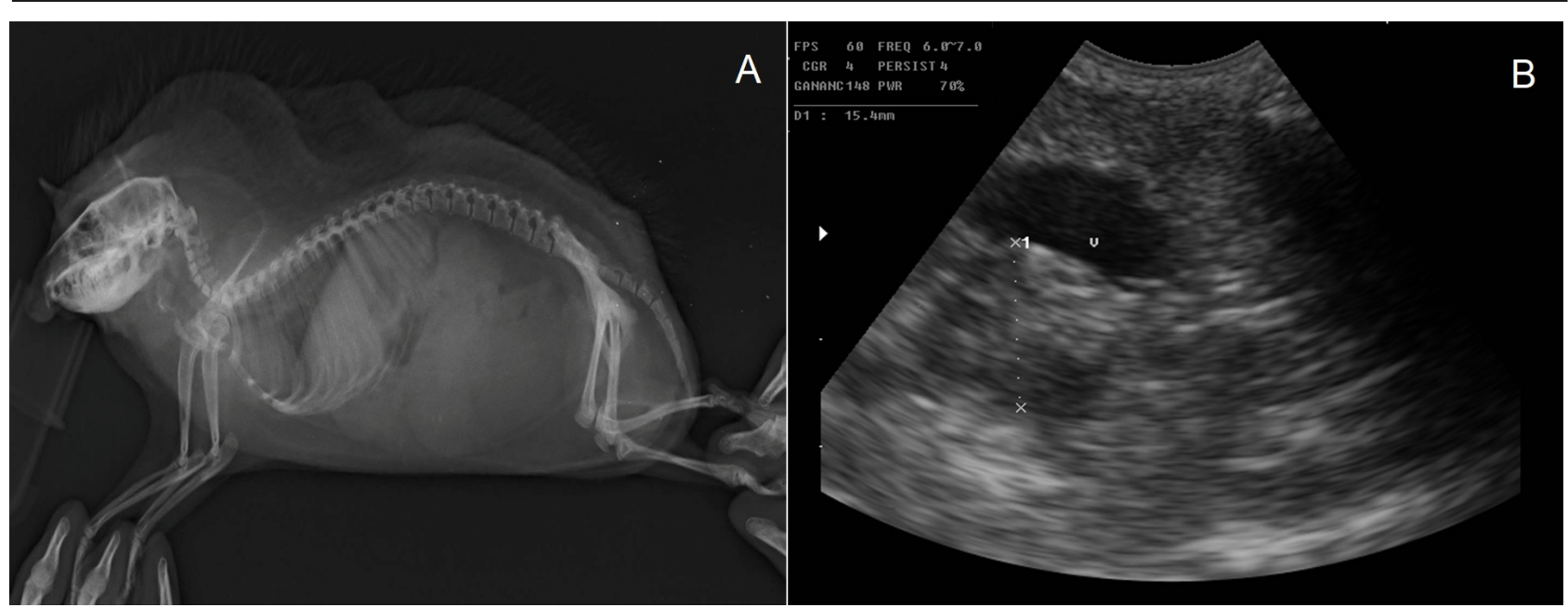

Figura 1. (A). Radiografía abdominal latero-lateral: vejiga pletórica sin urolitos radiopacos y aumento de la radiopacidad circunscripta en mesogastrio con desplazamiento de órganos vecinos, sugerente de masa. (B). Ecografía abdominal: neoformación intrauterina de 15,4 mm de diámetro. V: indica la vejiga urinaria.

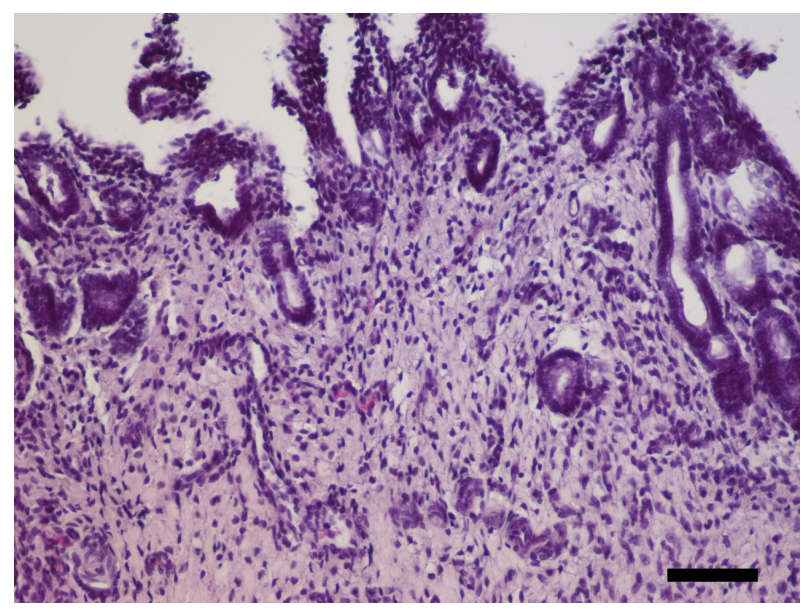

Figura 2. (A). Endometrio con edema e infiltrado mononuclear compuesto principalmente por macrófagos. H\&E, Barra $=100$ $\mu \mathrm{m}$.

La masa pedunculada estaba compuesta por tejido conectivo muy vascularizado, con fibroblastos, fibrocitos y macrófagos, y tapizada por células epiteliales normales (Figura 3A-B). La masa nodular estaba compuesta por abundante cantidad de células fusiformes, dispuestas en bandas entrelazadas, con un núcleo único, grande, central, redondo u oval con un patrón de cromatina en grumos y moderada cantidad de citoplasma. No se observaron figuras mitóticas (Figura 4A-B). Mediante la coloración especial de van Gieson se confirmó la presencia de abundantes fibras de musculo liso y escaso tejido conjuntivo (Figura 4C). Se formuló el diagnóstico de pólipo y leiomioma uterinos. Se otorgó el alta médico y quirúrgico a los 10 días de la intervención. La paciente se recuperó totalmente. Doce meses postintervención la misma concurrió a consulta para realizar un control anual en el cual se no se evidenciaron signos clínicos de la enfermedad.

\section{Discusión}

En los últimos años se ha comunicado el hallazgo de diversas neoplasias uterinas en el erizo pigmeo africano. Raymond \& Garner (2001) realizaron un estudio sobre erizos cautivos pudiendo observar que el 4,54 \% (3/66) padecía enfermedad neoplásica del útero, presentándose dos casos de leiomiosarcomas. En el trabajo realizado por Mikaelian \& Reavill, (2004) comunicaron que 15 erizos presentaban afecciones uterinas únicas o múltiples, observando 4 hembras con pólipos endometriales, 2 con pólipo endometrial y adenosarcoma y una hembra con adenoleiomiosarcoma conjuntamente con sarcoma del estroma endometrial. Done et al., (2007) comunicaron el caso de un erizo de 5 años de edad con hematuria debida a un pólipo endometrial y adenomiosis, siendo el tiempo de sobrevida de 19 meses.

En otro estudio pudo observarse que un erizo de 5 años presentó una masa en caudolateral del flanco izquierdo correspondiente a un osteosarcoma extraesquelético. Aproximadamente 2 meses después de la cirugía, el paciente murió repentinamente. Durante la necropsia pudo hallarse una masa uterina compatible con un leiomioma (Phair et al., 2011).

Tsai et al., (2016) observaron que un erizo de 2 años y 7 meses de edad presentó una masa subcutánea en lateral del cuello, así como una masa intraabdominal encontrada durante la palpación. Una vez realizada la remoción quirúrgica pudo observarse en el miometrio células neoplásicas, siendo el diagnóstico definitivo sarcoma de estroma endometrial y liposarcoma.

Okada et al., (2018) realizaron un estudio histológico de órganos reproductivos femeninos de erizos observando pólipos endometriales en 7 hembras $(n=33)$, siendo estos de presentación única o múltiple. Asimismo, los pólipos fueron pedunculados y ocupaban la luz uterina. Los 


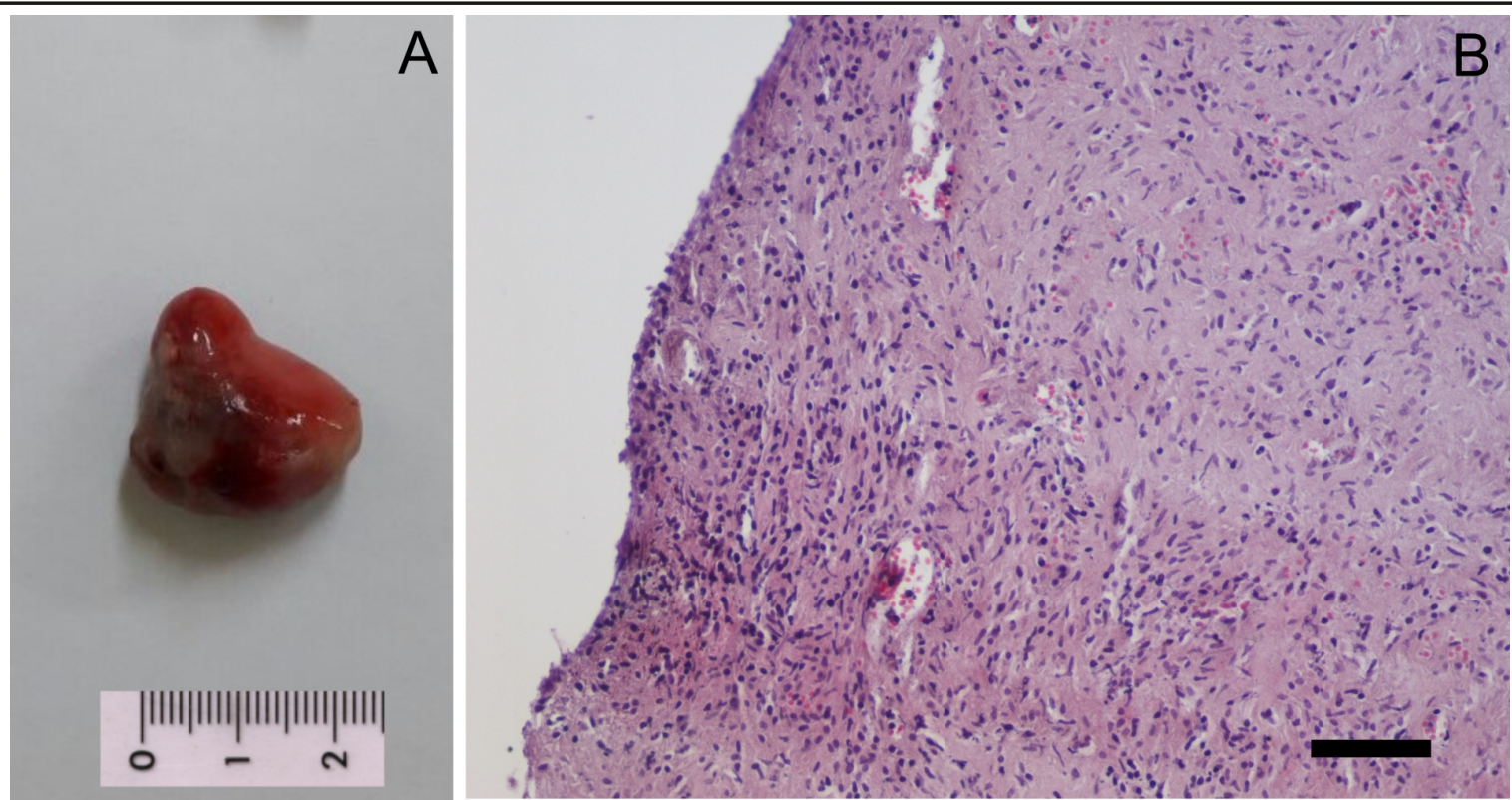

Figura 3. (A). Masa uterina pedunculada: Pólipo endometrial (B). Estroma muy vascularizado, con fibroblastos, fibrocitos y macrófagos. H\&E. Barra $=100 \mu \mathrm{m}$.

signos clínicos que se observaron fueron sangrado vaginal o hematuria (Okada et al., 2018).

Recientemente, Chambers et al. (2018) examinaron 50 úteros de erizos con signos clínicos de enfermedad uterina. Siete animales (14\%) presentaron pólipo endometrial y en $27(54 \%)$ se diagnosticó neoplasia endometrial. Al examen clínico la mayoría de las hembras presentaban hematuria o sangre adherida a la vulva. Las edades fueron 29,4-25,2 meses. Las neoplasias se clasificaron en 7 tumores mixtos endometriales, 12 nódulos del estroma endometrial y 8 sarcomas del estroma endometrial. Sin embargo, los nódulos estromales endometriales y los sarcomas del estroma endometrial a menudo fueron contiguos a un pólipo endometrial o a un tumor mixto. La tasa de supervivencia a los 180 días después de la ovario- histerectomía fue del $100 \%$ (4/4) en el pólipo endometrial y del $60 \%(3 / 5)$ en el tumor endometrial mixto, $100 \%(3 / 3)$ en nódulo estromal endometrial y 42,9 \% (3/7) en el sarcoma estromal endometrial.

Si bien se han realizado diversos estudios que comunican la alta prevalencia de neoformaciones uterinas y pólipos endometriales en el erizo pigmeo africano, nuestro trabajo es el primero en comunicar la presentación, en forma conjunta, de un pólipo endometrial y un leiomioma uterino. Asimismo, en la mayoría de los trabajos estas neoformaciones resultan ser un hallazgo de necropsia, mientras que en muy pocos trabajos consignan la sobrevida del animal luego de la remoción quirúrgica.
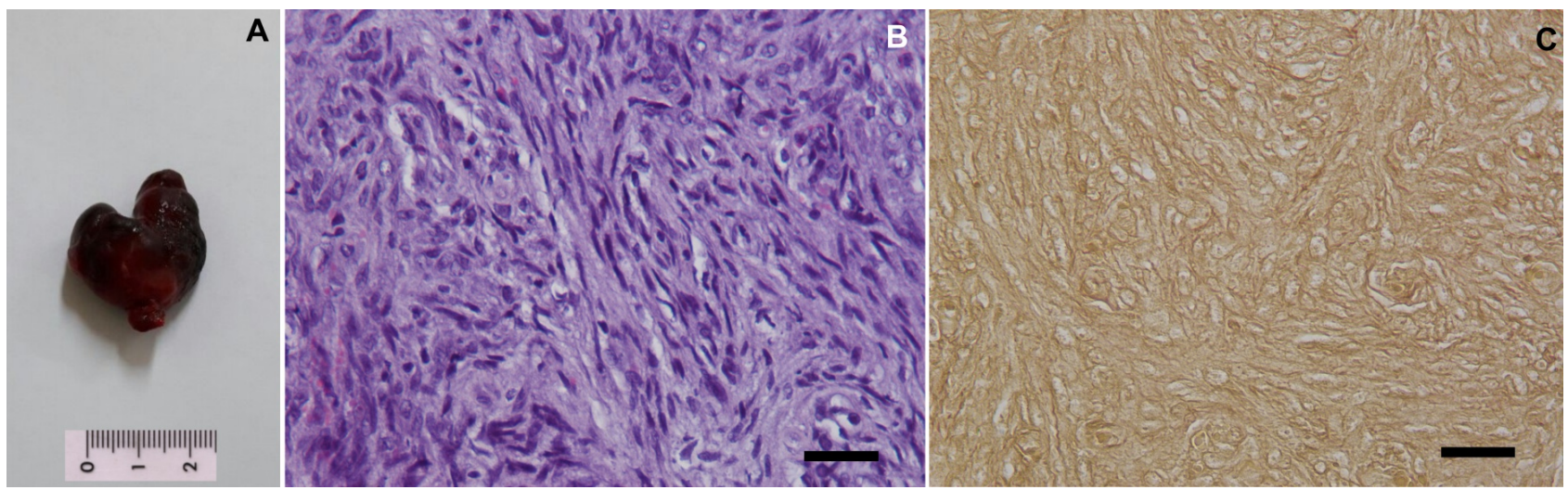

Figura 4. (A). Masa uterina nodular: Leiomioma (B). Abundante cantidad de células fusiformes, dispuestas en bandas entrelazadas. Las células presentan un núcleo único, grande, central, redondo u oval con un patrón de cromatina en grumos y moderada cantidad de citoplasma. H\&E. Barra $=50 \mu \mathrm{m}$. (C). Abundantes células musculares (amarillo) y escasas bandas de tejido conectivo (rojo). van Gieson. Barra $=50 \mu \mathrm{m}$. 


\section{Conclusiones}

La ovariohisterectomía es el tratamiento de elección para neoplasias uterinas en el erizo, lo cual permite aumentar su expectativa de vida. Asimismo, se debería recomendar a los propietarios de los erizos pigmeos africanos hembras que realicen controles períodicos reproductivos, con el fin de realizar un diagnóstico precoz de este tipo de afecciones.

\section{Agradecimientos}

Los autores agradecen al Servicio de Diagnóstico por Imágenes y al Servicio de Cirugía del Hospital Escuela de la Facultad de Ciencias Veterinarias de la Universidad Nacional de La Plata.

\section{Conflicto de intereses}

Todos los autores declaran que no existe conflicto de intereses, incluyendo las relaciones financieras, personales o de otro tipo con otras personas u organizaciones que pudieran influir de manera inapropiada en el trabajo.

\section{Bibliografía}

Chambers JK, Shiga T, Takimoto H, Dohata A, Miwa Y, Nakayama H, Uchida K. 2018. Proliferative lesions of the endometrium of 50 four-toed Hedgehogs (Atelerix albiventris). Veterinary Pathology 55(4):562-71.

doi: 10.1177/0300985818758467

Done LB, Deem SL, Fiorello CV. 2007. Surgical and medical management of a uterine spindle cell tumor in an African hedgehog (Atelerix albiventris). Journal of Zoo and Wildlife Medicine. 38(4):601-3.

doi: 10.1638/2006-0066R.1

Heatley JJ, Mauldin GE, Cho DY. 2005. A review of neoplasia in the captive African hedgehog (Atelerix albiventris). Seminars in Avian and Exotic Pet Medicine. 14(3):182-92.

doi: 10.1053/j.saep.2005.07.002

Kennedy PC. 1998. Histological classification of tumors of genital system of domestic animal. World Health Organization International
Histological Classification of Tumors of Domestic Animals. Second Series Vol. IV.

McEntee K, Nielsen SW. (1976). XVI. Tumours of the female genital tract. Bulletin of the World Health Organisation. 53(2-3):217-26.

Mikaelian I, Reavill DR. 2004. Spontaneous proliferative lesions and tumors of the uterus of captive African hedgehogs (Atelerix albiventris). Journal of Zoo and Wildlife Medicine. 216-20. doi:10.1638/01-077

Okada K, Kondo H, Sumi A, Kagawa Y. 2018. A retrospective study of disease incidence in African pygmy hedgehogs (Atelerix albiventris). The Journal of Veterinary Medical Science. 80(10): 1504-10. doi: 10.1292/jvms.18-0238

Phair K, Carpenter JW, Marrow J, Andrews G, Bawa B. 2011. Management of an extraskeletal osteosarcoma in an African hedgehog (Atelerix albiventris). Journal of Exotic Pet Medicine. 20(2): 151-5. doi: 10.1053/j.jepm.2011.02.011

Phillips ID, Taylor JJ, Allen AL. 2005 . Endometrial polyps in 2 African pygmy hedgehogs. Canadian Veterinary Journal. 46(6):524-7.

Raymond JT, Garner MM. 2001. Spontaneous tumours in captive African hedgehogs (Atelerix albiventris): a retrospective study. Journal of Comparative Pathology. 124(2-3):128-33. doi: 10.1053/jcpa.2001.0441

Santana EM, Jantz HE, Best TL. 2010. Atelerix albiventris (Erinaceomorpha: Erinaceidae) Mammalian Species. 42(857): 99-110.

doi: 10.1644/857.1

Schlafer DH, Miller, RB. Female genital system. En: Maxie, MG. 2007. Pathology of domestic animals. Elsevier Sanders, Philadelphia, Pennsylvania. vol. 3,5 th ed. 429-564.

Sohn GS, Cho S, Kim YM, Cho CH, Kim MR, Lee SR. 2018. Current medical treatment of uterine fibroids. Obstetrics \& Gynecology Science. 61(2): 192-201. doi: 10.5468/ogs.2018.61.2.192

Tsai FY, Chang HM, Chang HK, Kao JP, Liao JW. 2016. Case report: endometrial stromal sarcoma and liposarcoma in an African hedgehog (Atelerix albiventris). Taiwan Veterinary Journal. 42 (03): 181-6. doi: 10.1142/S1682648515720117 